\title{
On Prosody Intelligence and Pedagogy for EFL learners in China
}

\author{
Yunhong Hou ${ }^{1, \mathrm{a}}$ \\ ${ }^{1}$ Xinjiang University, Foreign Languages School, Urumqi 830046, Peoples R China. \\ a43604243@qq.com
}

Keywords: Prosody intelligence, Pedagogy, EFL.

\begin{abstract}
Prosody intelligence is vital for EFL learners. In order to teach how to speak fluent English with less foreign accent, this paper reports Chinese EFL learners' prosody intelligence and discusses the pedagogy to improve the accuracy of Chinese EFL learners' pronunciation.
\end{abstract}

\section{Introduction}

Prosody was originally a musical term and was later introduced into phonetics. A complete human language prosodic hierarchy includes the following units: moras, syllables, foot/phonological chunk, prosodic word, clitic group, adhesion of phonological phrases, intonation phrase and utterance. This paper reports on English prosodic features of China English language learners (stress, information focus, and pause) perception, starting from the prosodic features of English to English language speech prosody experiment design. According to some experimental results with the combination of the experimental study of rhythm teaching, we make an exploration of teaching English speech prosodic methods for Chinese learners.

\section{Foreign language learners' perception of prosodic features in English}

\subsection{The perception of information focus and pause}

Previous studies showed that: "learners information focus, the phrases that reported at the end of the sentence and the phrases at the end of a sentence and the time / place adverbial of prosodic features make the most difficult acquisition etc.." (Chen Hua, 2010). English is intonation language. In the language with unique tonality, intonation phrase is a part of the prosodic component structure expressed by phonology. Its definition is mainly semantic in nature, and it is the unit of information structure. Prominence in English syllable is also called the "stress". In acoustics the emphasis is usually achieved by pitch, intensity and duration characteristics. "Information focus" refers to one of the most prominent syllables in tone 1, also known as "pronounced accent" or "tone modulation"". "Pause" is the most common feature of boundary tone group. As it is in a tone group, the pause length is usually around 200 milliseconds or more, which can be perceived by listening.

\subsection{The perception of stressed syllables}

In the judgment of stress one can have the perception that obvious efforts lengthened pronunciation. Under normal circumstances, signs of English stress are pitch prominence or time intensity, for example. The students in the rules under the guidance of relatively easy to determine stress, but in the acquisition, often due to the influence of Chinese pronunciation, syllable sound is not correct, especially when the first syllable. This is caused by the lack of rhythmic structure in English teaching.

\section{English prosody teaching experiment}

In order to study the effectiveness of English prosody teaching, we conducted a semester long English prosodic experiment teaching freshmen of non-English major in Xinjiang University. 


\subsection{The research design}

First, the subjects were randomly selected, namely, a natural class was randomly selected from 86 College English classes in grade 17. There are 24 freshmen from non-English majors enrolled. Test and record his or her oral reading, and make a questionnaire survey. Two phonetic researchers rated the test results and sorted them according to the scores. According to the scores, 1 / 3 English phonetics ability was higher than the strong, middle and poor (8 each).Then, 24 students accepted the English prosody teaching course (12 weeks), focusing on stress, information focus and pause training, accounting for 2 / 3 of the total class hours.

Finally, test and record his or her oral reading (the same as the first test corpus), and also conduct a questionnaire survey.

\subsection{The research findings}

The first questionnaire survey shows that learners' knowledge of English prosody is vague and arbitrary. Prerecorded recordings also confirmed this feature.

The results of the second questionnaire survey show that learners' English prosody knowledge is obviously improved, and the position of stress and pause can be clearly stated, and the voice expression of focus information. The analysis of posttest recordings shows that learners' English prosody output has made significant progress.

\subsection{The speech instance}

A case study of the stress patterns of noun phrases in English learners with poor phonological competence. As is shown in figure 1 stress pattern diagram of learners' wide focus noun phrases

The stress of noun phrases in English native speakers is concentrated in the noun in the circle of the picture. Performance for the pitch contour is slightly higher than the surrounding syllables, highlighting the obvious characteristics. In pre-test, learners with poor English phonological competence have various types of stress. In the post test, the noun phrase stress of he (she) is concentrated in the noun in the circle in figure 1. This shows that learners can correctly grasp English stress patterns through English prosody teaching.

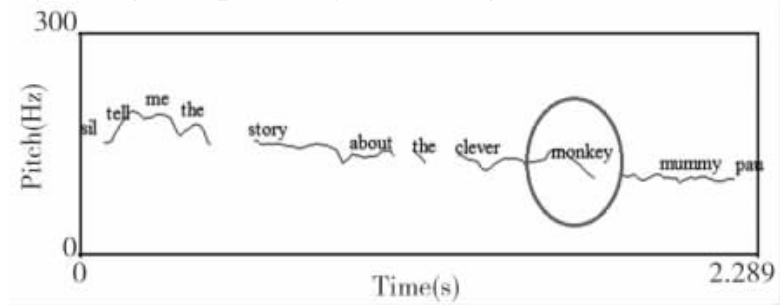

Figure 1 stress pattern diagram of learners' wide focus noun phrases

The stress of noun phrases in English native speakers is concentrated in the noun in the circle of the picture. Performance for the pitch contour is slightly higher than the surrounding syllables, highlighting the obvious characteristics. In pre-test, learners with poor English phonological competence have various types of stress. In the post test, the noun phrase stress of he (she) is concentrated in the noun in the circle in figure 1. This shows that learners can correctly grasp English stress patterns through English prosody teaching.

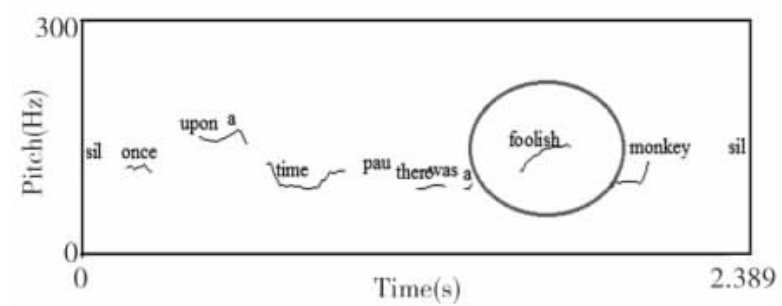

Figure 2 stress pattern of learners with narrow focus noun phrases 


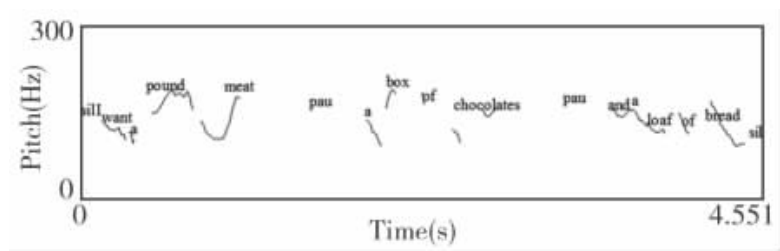

Figure 3 information focus stress pattern of learners

According to the core stress rules proposed by Chomsky and Halle (1968), the phrase stress is transferred from the original position to the contrastive feature position when there is a marked situation. At this time, the phonetic representation is at the same time the duration of pitch highlights. In pre-test, learners with poor English phonological competence have various types of stress. After the test, the noun phrases of his or her nouns are all stressed in the noun in the circle in figure 2. This shows that learners can correctly grasp English stress patterns through English prosody teaching.

\section{Conclusion}

It is relatively easy for the foreign language learners to acquire phrase word stress patterns of English, but once in the language flow the foreign accent is still widespread phenomenon. So most scholars believe that in speech prosody duration, pitch and intensity are three basic elements with pitch and intensity as the main factors to determine the nature of the English speech. In addition, rhythm and pause are also important factors that determine the naturalness of English pronunciation. Through listening experiments on China students English pronunciation, rhythm and pause errors is one of the main reasons of foreign accent. Therefore, English language teaching in the training of two words and phrases of pitch duration at the same time, more attention should be paid to training in the sentence rhythm and pause mode.

Language aptitude, like musical talent, varies from person to person, so language learners have different acquisition effects. Language gifted learners are few in number after all, which requires the involvement of professional English instructors and foreign language acquisition researchers to conduct systematic science guides for their language learning.

\section{Acknowledgement}

In this paper, the research was sponsored by the Ministry of Education Social Science Youth Fund for Xinjiang Uygur Autonomous Region of China (Project No. 17XJJC740001).

\section{References}

[1] Chen Hua, Wang Xinmin. A study on the characteristics of Chinese students' English phrase stress [J]. Foreign Language and Foreign Language Teaching,2015,(03):13-18+40.

[2] Manli Lu. A Corpus-Based Study on Semantic Prosody of English Synonyms "Common" and “Ordinary”[A]. Information Engineering Research Institute, USA. Proceedings of 2015 3rd International Conference on Education Reform and Management Innovation(ERMI 2015 V78)[C].Information Engineering Research Institute, USA:,2015:5.

[3] Na Li. EFL Peer Chinese-English Formulaic Sequence Translation Based on Prosody[A]. The International Science and Culture Center for Academic Contacts(ISCCAC)Huanghe College of Science and Technology(HCST). Proceedings of the International Conference on Education,Language,Art and Intercultural Communication(ICELAIC 2014) Part B[C].The International Science and Culture Center for Academic Contacts(ISCCAC)Huanghe College of Science and Technology(HCST):,2014:4.

[4] Shen Si. A Contrastive Study of Semantic Prosody between English Native Speakers and 
Chinese English Learners_-Case Study of “Avoid”[J]. Overseas English,2013,(01):240-241+257.

[5] Li Yinghao Linguistics Lab, Peking University. English Sentence Prosody Produced by Learners in China[A]. Proceedings of the 8th Chinese Phonetics Conference [C]. Language Institute of the Chinese Academy of Social Sciences, 2008:7. 